\title{
Equidade em saúde: Reflexões do princípio doutrinário potencializador da atenção primáría a saúde
}

\author{
Health equity: Reflections on the doctrinal principle that potentiates primary health care
}

Equidad en salud: Reflexiones sobre el principio doctrinal que potencia la atención primaria de salud

\begin{abstract}
RESUMO
Objetivo: analisar as contribuições do princípio equidade no contexto da Atenção Primária à Saúde, bem como assistência de enfermagem no âmbito da equidade em saúde. Método: Realizou-se um estudo de revisão bibliográfica de literatura na SciELO e LILACS, realizada no período de julho de 2020 a setembro de 2020, com os descritores em ciências da Saúde: "Atenção Primária à Saúde"; "Equidade em saúde" e "Sistema Único de Saúde", aplicando-se o operador "and", sendo os critérios de inclusão Língua Portuguesa, textos completos e publicados no período de 2015 a 2020. Como critérios de exclusão artigos de revisão, duplicados e que não contemplavam o objetivo. Resultados: Utilizando-se 13 artigos para o estudo. Discussão: A equidade é potencializadora do Sistema Único de saúde, na qual apresenta a resolutividade das ações e da assistência de enfermagem. Conclusão: A equidade reduz as desigualdades durante as práticas de saúde, proporcionando o cuidado pautado nas políticas públicas.
\end{abstract}

DESCRITORES: Atenção Primária à Saúde. Equidade em saúde. Sistema Único de Saúde.

\section{ABSTRACT}

Objective: to analyze the contributions of the equity principle in the context of Primary Health Care, as well as nursing assistance in the scope of health equity. Method: A literature review study was carried out in SciELO and LILACS, carried out from July 2020 to September 2020, with the descriptors in Health Sciences: "Primary Health Care"; "Equity in health" and "Unified Health System", applying the "and" operator, with the inclusion criteria Portuguese Language, complete texts and published in the period from 2015 to 2020. As exclusion criteria, duplicate review articles and that did not contemplate the objective. Results: Using 13 articles for the study. Discussion: Equity is an enhancer of the Unified Health System, in which it presents the resoluteness of actions and nursing care. Conclusion: Equity reduces inequalities during health practices, providing care based on public policies. DESCRIPTORS: Primary Health Care. Health equity. Health Unic System.

\section{RESUMEN}

Objetivo: analizar los aportes del principio de equidad en el contexto de la Atención Primaria de Salud, así como la asistencia de enfermería en el ámbito de la equidad en salud. Método: Se realizó un estudio de revisión de la literatura en SciELO y LILACS, realizado de julio de 2020 a septiembre de 2020, con los descriptores en Ciencias de la Salud: "Atención Primaria de Salud"; "Equidad en salud" y "Sistema Único de Salud", aplicando el operador "y", con los criterios de inclusión Lengua Portuguesa, textos completos y publicados en el período de 2015 a 2020. Como criterio de exclusión, artículos de revisión duplicados y que no contemplaba el objetivo. Resultados: Utilizando 13 artículos para el estudio. Discusión: La equidad es un potenciador del Sistema Único de Salud, en el que presenta la resolución de las acciones y los cuidados de enfermería. Conclusión: La equidad reduce las desigualdades durante las prácticas de salud, brindando atención basada en políticas públicas.

DESCRIPTORES: Atención Primaria de Salud; Equidad en salud; Sistema único de Salud.

RECEBIDO EM: 15/09/2020 APROVADO EM: 22/10/2020

\section{Brenda Pinheiro Evangelista}

Graduanda do Curso de Bacharelado em Enfermagem no Centro Universitário Vale do Salgado.

ORCID 0000-0002-8830-2681

\section{Rafael Bezerra Duarte}

Enfermeiro. Docente do curso de Bacharelado em Enfermagem no Centro Universitário Vale do Salgado. Mestrando em Saúde Coletiva pela Universidade Estadual do Ceará.

ORCID: 0000-0002-2280-0864. 


\section{artigo}

Evangelista, B.P.; Duarte, R.B.; Freitas, L.M.; Evangelista, B.P.; Oliveira, M.E.L.; Xavier Silva, J.P.

Equidade em saúde: Reflexões do princípio doutrinário potencializador da atenção primária a saúde

\section{Kerma Márcia de Freitas}

Coordenadora do curso de Bacharelado em Enfermagem no Centro Universitário Vale do Salgado. Mestre em Saúde Coletiva e Doutoranda em Saúde Coletiva pela UNIFOR.

ORCID: 0000-0003-3021-4758

\section{Breno Pinheiro Evangelista}

Graduando do Curso de Bacharelado em Farmácia na Faculdade São Francisco da Paraíba. ORCID: 0000-0001-6881-0980

\section{Maria Edméa Lopes de Oliveira}

Graduanda do curso de Bacharelado de Enfermagem do Centro Universitário Vale do Salgado.

ORCID: 0000-0002-6994-6625

\section{João Paulo Xavier Silva}

Enfermeiro. Mestre em Enfermagem. Docente do Curso de Bacharelado em Enfermagem no Centro Universitário Vale do Salgado.

ORCID: 0000-0003-3082-9373

\section{INTRODUÇÃO}

0 termo equidade pode estar associado à igualdade, tendo em vista que ambas se referem à superação das desigualdades econômicas e sociais. No que concerne à saúde, ela constitui-se com ênfase na avalição do controle social, eficiência, efetividade e participação de reformas setoriais, para a reorientação de políticas e de sistemas de saúde ${ }^{10}$.

Nesse sentido, a equidade associada ao contexto da saúde apresenta-se diante do pressuposto de que a distribuição de recursos deve ser realizada de acordo com as necessidades que um determinado grupo ou população apresenta, e no sentido de tratar cada usuário mediante a priorização no atendimento, critérios clínicos, sociais e epidemiológicos, aos que mais necessitam ${ }^{12}$.

A equidade está relacionada à duas vertentes, sendo elas: o tratamento das pessoas iguais de forma igual, onde promova a universalidade e a igualdade diante do acesso. Já tratar os desiguais de forma desigual caracteriza-se como a segunda vertente, interligada à prioridade as pessoas de grupos específicos, mediante os critérios de riscos e que são mais necessitadas de recursos socioeconômicos ${ }^{1}$.

Deste modo, a equidade em saúde contempla superar a falta de disparidades, seguida de uma avaliação crítica concernente às vantagens e desvantagens da sociedade associadas à acessibilidade e aos cuidados referentes a saúde, com uma redistribuição imposta a quem tem menos e a quem apresenta as mesmas condiçóes, partindo de uma ação que regule as desigualdades ${ }^{8}$.

Desta forma, considerando a equidade como um dos principais pilares no contexto do Sistema Único de Saúde (SUS), faz-se possível a ampliação de esforços para que aconteça a redução significativa dos determinantes, condicionantes e agravos em saúde, de acordo com a compreensão das diferenças, como etnia, religião, orientação sexual, identidade de gênero, posição socioeconômica, educação, condições de saúde e situações de marginalização social que influenciam na qualidade de vida ${ }^{6}$.

Embora a Lei no 8.080/1990, que regulamenta o Sistema Único de Saúde, apresente o princípio da igualdade, não enfatizando equidade, faz-se necessário ressaltar a sua contextualização exposta nos documentos oficiais da Federação durante a Norma Operacional Básica de 1996 (NOB-96), bem como no capítulo da Seguridade Social da Constituição Brasileira de 1988, onde apresenta o financiamento e distribuição de recursos na saúde, de acordo com o princípio da equidade ${ }^{3}$.

A universidade, a integralidade e a equidade são os princípios doutrinários do SUS, os quais garantem o direito à saúde de forma ampla e integral, sendo a equidade fundamental para a promoção da saúde a partir do direito à cidadania no acesso aos serviços de saúde. Ela constitui- -se como um princípio precursor da superação de desigualdades e favorece a justiça que, por sua vez, promove o atendimento aos usuários mediante as suas necessidades por meio do reconhecimento das diferenças e condiçôes de saúde ${ }^{2}$.

Nessa perspectiva, a equidade proporciona uma visão diferenciada aos grupos populacionais que necessitam de prioridades que precisam, fazendo-se notória a implementação desse princípio para a efetivação de políticas públicas e promoção da saúde, contribuindo para o cuidado integral e humanizado para as pessoas?.

A equidade se encontra relacionada com os serviços da Atenção Primária à Saúde (APS) por proporcionar a ampliação do acesso associado às necessidades dos usuários. Nesse contexto, a acessibilidade no âmbito primário contribui para a efetivação da equidade e para os avanços na saúde pública, promoção da saúde e regulamentação dos serviços existentes na atenção básica ${ }^{4}$.

Deste modo, a equidade é considerada um ponto forte para a ampliação do acesso universal em saúde e redução das desigualdades. Vale destacar que esse princípio é fundamental nos serviços da atenção básica, uma vez que contribui para a superação das vulnerabilidades em saúde e para potencializar as políticas públicas ${ }^{6}$.

Nessa conjuntura, percebendo a importância do principio da equidade no que diz respeito as práticas em saúde, ques- 
tiona-se: quais as contribuições da equidade durante a assistência de enfermagem na Atenção Primária à Saúde?

$\mathrm{O}$ interesse pela temática teve como motivação a participação do pesquisador em um curso de aperfeiçoamento intitulado "Promoção da equidade no SUS", oferecido pela Universidade Aberta do Nordeste da Fundação Demócrito Rocha em parceria com a Universidade Estadual do Ceará. Como contribuição, têm-se a assistência, ensino e a pesquisa. Acredita-se que refletir os elementos teórico-conceituais da equidade seja um dos caminhos para aprimorar a assistência conferida nos serviços de atenção a grupos considerados vulneráveis.

O estudou buscou analisar as contribuições do princípio equidade no con- texto da Atenção Primária à Saúde, bem como a assistência de enfermagem no âmbito da equidade em saúde.

\section{MÉTODO}

Trata-se de um estudo de revisão bibliográfica de literatura, realizada no período de julho de 2020 a setembro de 2020, onde buscou-se discutir estudos no campo da enfermagem que contemplassem a temática voltada a equidade em saúde. Foi realizado uma busca nas seguintes bases de dados: Scientific Eletronic Library Online (SciELO) e Literatura Latino-americana e do Caribe em Ciências da Saúde (LILACS), com os descritores em ciências da Saúde: "Atenção Primária à Saúde”; "Equidade em saú- de" e "Sistema Único de Saúde", aplicando-se o operador "and", sendo os critérios de inclusão Língua Portuguesa, textos completos e publicados no período de 2015 a 2020. Como critérios de exclusão artigos de revisão, duplicados e que não contemplavam o objetivo da pesquisa. Foi utilizado o instrumento Ursi para a coleta de dados. Foi utilizado o instrumento Ursi para a coleta de dados.

\section{RESULTADOS}

Mediante a busca pelos artigos, foram encontradas 128 produções cientificas a partir dos critérios de inclusão, foram excluídos 96 em virtude de não se correlacionarem ao interesse temático, sendo utilizados 12 artigos para a discussão.

\section{Tabela 1 - Resultados dos estudos dos anos de 2015 a 2020, título, autor/ano e método.}

\section{Titulo}

Equidade: seus conceitos, significações e implicações para o SUS.

Aspectos bioéticos da judicialização da saúde por medicamentos em 13 municípios no meio Oeste de Santa Catarina, Brasil

Atenção Primária à Saúde: elementos de continuidade e mudanças na saúde do Distrito Federal.

Expansão da Atenção Primária à Saúde no Município do Rio de Janeiro em 2010: o desafio do acesso e a completude das equipes de saúde da familia.

Estado e políticas de equidade em saúde: democracia participativa?

Práticas de cuidado da equipe da estratégia saúde da família.

Um olhar sobre sistemas de saúde locais e nacionais.

O desafio da equidade: fatores associados aos gastos com a produção ambulatorial na atenção secundária.

Determinantes sociais, equidade e consultório na rua

Acesso ao cuidado na Atenção Primária à Saúde brasileira: situação, problemas e estratégias de superação

\author{
Autor \\ DE BARROS, F.P.C.; DE \\ SOUZA, M.F. \\ Ano \\ 2016
}

ZAGO B, SWIECH LM, BONAMIGO, EL,SCHLEMPER JUNIOR, BR.

KASHIWAKURA HK,GONÇALVES AO, DA SILVA, RM P.

2016 SIMÕES P.P, GAMEIRO F, NUNES PC, SILVA JÚNIOR AG, SCARDUA MT, KEITH BF.

SOUTO, K.M.B, SENA AGN, PEREIRA VOM, SANTOS, LM.

COUTO T. A, SANTOS FPA, ACIOLI S; MACHADO JC; MS, RODRIGUES VP, COUTO TA.

DE PAULA JB, IBIAPINA F, MOSSÉ P.

2016

2016

2016

2018

2018

LIEBEL G, SÁ JUNIOR AR, MÁRCIA E, CAMPOS S.

2018

PINTO AH, FERMO VC, PEITER CC, FERNANDES VMB, HEIDEMAN ITSB.

2018

TESSER C.D, NOR-MAN AH, VIDAL TB.

2018
Tipo de Estudo

Ensaio

Estudo quantitativo

Estudo documental

Estudo sobre a expansão da $A B$, em especial as equipes de saúde da família, no Município do Rio de Janeiro

Ensaio

Estudo qualitativo

Estudo Reflexivo

Estudo ecológico, analítico observacional

Estudo qualitativo, descritivo, tipo relato de experiência Estudo reflexivo 


\section{Análise de equidade da distribuição de recursos do} Programa Farmácia Popular

Desafios para a Educação Permanente em Saúde Indígena: adequando o atendimento do Sistema

Único de Saúde no sul do Brasil.

Desafios da enfermagem na promoção de práticas de equidade em saúde: um diálogo entre a enfermagem no Brasil e no Canadá
SILVA MEL, DE ALMEIDA ATC, ARAGÃO JÚNIOR ITA.
2019
Estudo transversal
LANDGRAF J, IMAZU NE, ROSADO RM.
2020
Relato de experiência
VELLOSO, I.S.C, PEREIRA MS, SALAS AS, ARAÚJO MT.
Estudo reflexivo

\section{DISCUSSÃO}

O princípio doutrinário denominado equidade é primordial para o progresso do SUS, em virtude do reconhecimento das diferenças acerca das condições de saúde e necessidades dos usuários, promovendo o direito à saúde e as diferenciações sociais mediante as diversidades. A equidade é relevante em todos os âmbitos referentes a assistência à saúde, uma vez que proporciona o estabelecimento de critérios para a avaliação do usuário para nortear o atendimento com base nas políticas públicas equitativas?

Esse princípio apresenta relação com a contextualização da igualdade bem como a justiça no âmbito da saúde, mediante o atendimento realizado de acordo com as necessidades dos indivíduos no qual disponibiliza os recursos, principalmente, para quem mais necessita. Desta forma, é perceptível a contribuição desse princípio no que diz respeito as práticas de saúde eficazes e com resolutividades ${ }^{12}$.

Nesse sentido, a equidade é precursora da promoção da saúde, sendo também considerada como eixo central para a superação de desigualdades e desvantagens sociais prevalentes nos usuários do SUS, onde o respeito às características socioeconômica, da família, da pessoa e condições favoráveis de moradia é uma realidade evidente diante dos pressupostos dessa política ${ }^{14}$.

A ampliação do acesso aos serviços de saúde encontra-se associada à garantia de todos os diretos à saúde por meio do SUS, sendo a equidade considerada um dos pilares desse sistema no que concerne a distribuição dos recursos. Assim, diante desse cenário referente ao acesso aos serviços provenientes da efetividade desse princípio, a expansão das açốes de saúde é um fenômeno a ser destacado ${ }^{6}$.
Em virtude das necessidades distintas de cada pessoa, as desigualdades devem ser superadas para que a assistência qualificada seja atribuída a população em geral. Nesse contexto, destaca-se o fortalecimento das ações do SUS amparadas e norteadas pelo princípio da equidade para a superação dos desafios que limitam o acesso e promovem a desigualdade nos recursos ${ }^{11}$.

Nesse contexto, a promoção e proteção da saúde são atributivas e potencializadas pelas ações do SUS por intermédio da reorganização dos serviços, sendo a equidade protagonista desse cenário. Ressalta-se, ainda, a participação desse princípio nos avanços nas redes de atenção e a coordenação do fortalecimento do cuidado, além de corroborar com a garantia do processo de territorialização, mapeamento das situações de riscos e vulnerabilidade social ${ }^{17}$.

A assistência de enfermagem contribui para a valorização da equidade no âmbito da saúde em diferentes níveis de atenção, mediante a criação de novos instrumentos de comunicação, tecnologias em saúde para ampliar o acesso, trabalho em equipe e estratégias voltadas a equidade, justiça social e políticas públicas para superar as dificuldades e desigualdades ${ }^{16}$.

O enfermeiro vem ampliando o seu papel referente a autonomia na tomada de decisões frente a identificação das necessidades e cuidados da população, bem como na formulação de estratégias que promovem estratégias de promoção e proteção da saúde em diferentes dimensões, constituindo-se como um componente fundamental nos sistemas de saúde?

É pertinente ressaltar que a reorientação do modelo assistencial materializado pela atenção primária a saúde e proporcionada pela configuração de equipes multiprofissionais em unidades básicas de saúde, com ênfase no enfermeiro, abrange mudanças significativas nos indicadores de saúde das pessoas assistidas, sendo primordial que esse profissional possa conhecer a realidade e intervir nos aspectos de saúde relacionados a vulnerabilidade, acessibilidade e iniquidades ${ }^{13}$.

O enfermeiro deve buscar subsídios e debates centrais que organizem as práticas de saúde com foco na equidade no acesso aos serviços de saúde, que promovam uma visão ampliada para a superação dos desafios em diferentes dimensões, como na assistência e na gestão ${ }^{14}$.

A literatura enfatiza que o profissional da enfermagem deve assumir o seu protagonismo e a responsabilidade para promover a inclusão e o respeito as diferenças para o acesso equânime de forma justa e inclusiva. Vale ressaltar que os profissionais da enfermagem também apresentam a posição de educadores no que concerne a ampliação do conhecimento da sociedade sobre a equidade em saúde ${ }^{15}$.

Ademais, aponta-se que os enfermeiros enfrentam desafios para realizar e aprimorar a assistência equânime, no sentido de prevenir e corrigir as iniquidades em saúde que afetam a população, estando esses desafios relacionados a falta de mecanismos que possam ampliar e acelerar o desenvolvimentos das estratégias das políticas de saúde existentes para promover mudanças significativas ${ }^{16}$.

Embora o enfermeiro seja considerado um agente central no processo da assistência para promoção da equidade no cenário da atenção primária, os desafios do cotidiano e a sobrecarga de trabalho impedem a reformulação de novas estratégias que avancem nesse processo, fazendo-se necessária a elaboração de novas ações referentes ao acolhimento e ampliação para o acesso ${ }^{4}$. 
A utilização dos serviços de saúde por meio da assistência de enfermagem encontra-se relacionada diretamente com a disponibilidade referente à oferta de serviços, acessibilidade em virtude da proximidade e/ou facilidade geográfica do serviço, organização do trabalho para atenção, fluxos, horários, rotinas, prioridades técnicas, aceitabilidade relacionada a compreensão e aceitação com as características da população, ou seja, suas crenças, condições socioeconômicas e satisfação com o serviço utilizado ${ }^{13}$.

Nessa perspectiva, a assistência de enfermagem pode ampliar o acesso equânime e universal à saúde por meio da redução de desigualdades associadas à política pública prioritária que pressupóe a garantia de acesso à rede ambulatorial e domiciliar em diferentes níveis de complexidade mediante as necessidades das pessoas assistidas ${ }^{6 ; 8}$.

É evidente a contribuição da assistência de enfermagem diante desse cenário, no qual a ampliação de ações coletivas e o desenvolvimento de práticas integradoras do cuidado favorece novas possibilidades relacionadas a superação das vulnerabilidades sociais e dificuldades para o acesso aos serviços de saúde e proporcionar a promoção e proteção da saúde e prevenção de agravos 5 .

\section{CONCLUSÃO}

Portanto, a equidade incorpora a redu- ção das desigualdades por meio da efetivação dos elementos norteadores do SUS, que se dá potencialmente no cenário de práticas da APS Conforme a necessidade dos usuários são realizadas ações que conduzem práticas e ações de saúde, no sentido de efetivar o cuidado e a resolutividade dos serviços conduzidos. Vale ressaltar que a assistência de enfermagem pautada nas políticas públicas associadas à equidade desde o surgimento do SUS vem de encontro com as necessidades dos usuários, onde se busca a elaboração de estruturas transversais e recortes setoriais, além de promover ações para grupos em situação de vulnerabilidade que superem as desigualdades no contexto da APS. -

\section{REFERÊNCIAS}

1. Mercante JIS, Rodrigues RG, Alvarenga MT, Kraievski ES. HPV e sua influência no câncer de colo de útero. Rev Conexão Eletrônica. 2017;14(1):182-189. Available from: http://revistaconexao. aems.edu.br/wp-content/plugins/download-attachments/includes/download.php?id=77

2. Ministério da Saúde (BR), Secretaria de Vigilância em Saúde, Coordenação-Geral do Programa Nacional de Imunizações. Informe técnico sobre a vacina papilomavírus humano (HPV) na atenção básica [Internet]. Brasília: Ministério da Saúde; 2014 [cited 2020 may 12]. Available from: https://portalarquivos2. saude.gov.br/images/pdf/2015/junho/26/Informe-T--cnico-Introdu----o-vacina-HPV-18-2-2014.pdf

3. Ministério da Saúde (BR), Secretaria de Vigilância em Saúde. Boletim Informativo do PNI-02/2016 - Vacinação contra HPV [Internet]. Brasilia: Ministério da Saúde; 2016 [cited 2020 may 06]. Available from: https://www.conasems.org.br/wp-content/uploads/2016/10/ images_Boletim_informativo__HPV002-2016.pdf

4. Ministério da Saúde (BR). Ministério da Saúde convoca 10 milhões de adolescentes para vacinação de HPV e meningite [Internet]. Brasília: Ministério da Saúde; 2018 [cited 2020 may 06]. Available from: http://portalarquivos2.saude.gov.br/images/ pdf/2018/marco/13/Campanha-HPV-2018.pdf

5. Pinheiro C. O que afasta os jovens da vacina contra o HPV [Internet]. Veja Saúde; 2017 [cited 2019 set 19]. Available from: https://saude.abril.com.br/medicina/jovens-vacina-do-hpv/

6. Miot HA. Tamanho da amostra em estudos clínicos e experimentais. J Vasc Bras. 2011;10(4):275-278. Available from: https://www.scielo.br/pdf/jvb/v10n4/v10n4a01

7. Ministério da Saúde (BR), DATASUS, SIPNI - Sistema de Informações do Programa Nacional de Imunizações. Ministério da Saúde; 2018. Available from: <http://sipni-gestao.datasus.gov. br/si-pni-web/faces/relatorio/consolidado/dosesAplicadasCampanhaMultivacinacaoAdolescentes.jsf>.
8. Organização Pan-Americana da Saúde. Metodologia para o cálculo de cobertura da vacina contra o HPV na Região das Américas [Internet]. Washington, D.C.: OPAS; 2019. Available from: https://www.paho.org/pt/documents/methodology-calculate-hpv-vaccine-coverage-region-americas

9. Ministério da Saúde (BR), Conselho Nacional de Saúde. Resolução $n^{\circ}$ 466, de 12 de dezembro de 2012 [Internet]. Brasília: Ministério da Saúde; 2012 [cited 2020 jul 01] Available from: http://conselho.saude.gov.br/resolucoes/2012/Reso466.pdf

10. Kreuger MRO, Lizott LS, Friedrich HA. Imunização contra HPV: nível de conhecimento dos adolescentes. Adolesc Saude. 2017;14(3):38-45 [cited 2020 jun 21]. Available from: https://cdn. publisher.gn1.link/adolescenciaesaude.com/pdf/v14n3a06.pdf

11. Reiter PL, Katz ML, Paskett ED. Correlates of HPV vaccination among adolescent

12. females from appalachia and reasons why their parents do not intend to vaccinate. Rev Vaccine. 2013;(31):3121-3125. Available from: doi:10.1016/j.vaccine.2013.04.068

13. Moura LL. Cobertura vacinal contra o Papilomavírus Humano (HPV) em meninas e adolescentes no Brasil: análise por coortes de nascimentos [dissertação]. Rio de Janeiro: Fundação Oswaldo Cruz, Escola Nacional de Saúde Pública Sergio Arouca; 2019.

14. Kornides ML, McRee AL, Gilkey MB. Parents who decline HPV vaccination: who later accepts and why? Acad Pediatr, review. 2018;18(2):37-43 [cited 2020 june 28]. DOI: https://dx.doi. org/10.1016\%2Fj.acap.2017.06.008

15. Zanini NV, Prado BS, Hendges RC, Santos CA, Rodovalho-Callegari FV, Bernuci MP. Motivos para recusa da vacina contra o Papilomavírus Humano entre adolescentes de 11 a 14 anos no município de Maringá-PR. Rev Bras Med Fam Comunidade. 2017;12(39):1-13. DOI: http://dx.doi.org/10.5712/rbmfc12(39) 1253 